\title{
Estratégia Saúde da Família: caracterização dos usuários com diagnóstico de Saúde Mental
}

\author{
Family Health Strategy: characterization of users diagnosed with Mental Health \\ Estrategia Salud de la Familia: caracterización de usuarios diagnosticados con Salud Mental
}

Recebido: 12/01/2021 | Revisado: 14/01/2021 | Aceito: 15/01/2021 | Publicado: 18/01/2021

\author{
Aline Josiane Waclawovsky \\ ORCID: https://orcid.org/0000-0003-2990-4516 \\ Universidade Federal de Santa Maria, Brasil \\ E-mail: alinejw@yahoo.com.br \\ Alessandra Luana Schwantes \\ ORCID: https://orcid.org/0000-0002-8292-9782 \\ Universidade Federal de Santa Maria, Brasil \\ E-mail: als.fono@gmail.com \\ Ana Eliza Belizario Rodrigues \\ ORCID: https://orcid.org/0000-0001-7740-807X \\ Universidade Federal de Santa Maria, Brasil \\ E-mail: anaelizabelizario@gmail.com \\ Fabiana Einloft \\ ORCID: https://orcid.org/0000-0003-3630-1908 \\ Universidade Federal de Santa Maria, Brasil \\ E-mail: fabianaeinloft@gmail.com \\ Caren Fabiana Alves \\ ORCID: https://orcid.org/0000-0001-6911-8195 \\ Prefeitura Municipal de Santa Maria, Brasil \\ E-mail: carenfabi@gmail.com \\ Bruna Marta Kleinert Halberstadt \\ ORCID: https://orcid.org/0000-0002-4936-6156 \\ Universidade Federal do Pampa, Brasil \\ E-mail: enfermagembruna21@gmail.com \\ Teresinha Heck Weiller \\ ORCID: https://orcid.org/0000-0003-2531-0155 \\ Universidade Federal de Santa Maria, Brasil \\ E-mail: weiller2@hotmail.com
}

\begin{abstract}
Resumo
Objetivo: caracterizar os usuários com diagnóstico de saúde mental que acessam uma Estratégia Saúde da Família em um município do estado do Rio Grande do Sul. Metodologia: estudo transversal e descritivo. Após a coleta de dados, procedeu-se a análise estatística descritiva. Resultados: a amostra foi composta por 266 prontuários. A média de idade dos usuários foi de 59,07 anos, sendo 80,5\% do sexo feminino. A depressão foi o diagnóstico mais prevalente, seguido de transtorno de ansiedade. Predominam usuários solteiros (13,2\%), 40,6\% moram com familiares e/ou com o (a) companheiro (a), 38,7\% tinham ensino fundamental incompleto, 77,1\% faziam uso de antidepressivos e 50,4\% de benzodiazepínicos, 15,4\% utilizam dois antidepressivos diferentes, 1,5\% dois anticonvulsivantes e 2,3\% dois antipsicóticos. Conclusão: a falta de informação nos prontuários foi identificada, o que limitou a caracterização dos usuários. A depressão foi o diagnóstico mais prevalente, situação que a reforça como um importante problema de saúde pública.
\end{abstract}

Palavras-chave: Atenção primária à saúde; Estratégia saúde da família; Saúde mental.

\begin{abstract}
Objective: to characterize users with mental health diagnosis who access a Family Health Strategy in a city in the state of Rio Grande do Sul. Methodology: cross-sectional and descriptive study. After data collection, descriptive statistical analysis is performed. Results: the sample consisted of 266 medical records. The average age of users was 59.07 years, with $80.5 \%$ being female. Depression was the most prevalent diagnosis, followed by anxiety disorder. Single users predominate (13.2\%), $40.6 \%$ live with family and / or partner, $38.7 \%$ had incomplete primary education, $77.1 \%$ used antiepressants and 50.4\% benzodiazepines, $15.4 \%$ use two different antidepressants, $1.5 \%$ two anticonvulsants and 2.3\% two antipsychotics. Conclusion: the lack of information in the medical records was identified, limited the characterization of users. Depression was the most prevalent diagnosis, a situation that reinforces it as an important public health problem.
\end{abstract}

Keywords: Primary health care; Family health strategy; Mental health. 


\section{Resumen}

Objetivo: caracterizar a los usuarios con diagnóstico de salud mental que acceden a una Estrategia de Salud de la Familia en una ciudad del estado de Rio Grande do Sul. Metodología: estudio transversal y descriptivo. Después de la recolección de datos, se realizó un análisis descriptivo. Resultados: la muestra estuvo conformada por 266 historias clínicas. La edad media de los usuarios fue de 59,07 años, siendo el 80,5\% mujeres. La depresión fue el diagnóstico más prevalente, seguida del trastorno de ansiedad. Predominan los usuarios solteros (13,2\%), el 40,6\% vive con familiares y / o pareja, el 38,7\% tenía educación básica incompleta, el 77,1\% usa antidepresivos y el 50, 4\% benzodiazepinas, $15,4 \%$ utiliza dos antidepresivos diferentes, $1,5 \%$ dos anticonvulsivos y 2,3\% dos antipsicóticos. Conclusión: se identificó la falta de información en las historias clínicas, lo que limitó la caracterización de los usuarios. La depresión fue el diagnóstico más prevalente, situación que la refuerza como un importante problema de salud pública. Palabras clave: Atención primaria de salud; Estrategia de salud familiar; Salud mental.

\section{Introdução}

A saúde mental é compreendida como um processo complexo e desafiador sob a ótica dos trabalhadores de saúde dos diferentes serviços, gerando incessantes discussões no âmbito nacional e internacional, principalmente no que se refere ao perfil e a caracterização dos usuários que apresentam transtornos mentais. O cuidado em saúde mental envolve a inter-relação entre a equipe multiprofissional, usuário, família, comunidade e os coordenadores da gestão dos serviços que constituem a Rede de Atenção à Saúde (RAS) (Sampaio, et. al., 2011). Neste sentido, a Atenção Primária à Saúde (APS) é considerada a porta de entrada preferencial e a ordenadora das redes de atenção, em especial para a saúde mental, visto que a atuação no território é de responsabilidade da equipe da APS (Silva, et. al., 2015).

Considerar os princípios doutrinários do Sistema Único de Saúde (SUS) é essencial para a garantia do cuidado em saúde mental, assim como a universalidade, conforme descrito na Lei $\mathrm{n}^{\circ}$ 8.080/90, determina que todo cidadão, sem discriminações, tem direito de acesso aos serviços de saúde, visto que é de responsabilidade dos serviços locais garantir a assistência integral aos usuários de saúde mental (Silva, Moreira \& Aguiar, 2016). Neste contexto, a APS, por meio da Estratégia Saúde da Família (ESF), configura-se como um campo potencial de práticas e de promoção de novos modos de cuidado em saúde mental, tendo como objetivo atuar por meio do cuidado integrado e humanizado, orientado pela interdisciplinaridade, intersetorialidade e a territorialidade (Oliveira, et. al., 2017).

Segundo Oliveira et. al. (2014) a Portaria 3.088/2011, que institui a Rede de Atenção Psicossocial (RAPS), estabelece que a APS deve ser responsável em promover ações de promoção em saúde mental, prevenção de doenças e agravos, acompanhamento dos usuários que possuem diagnóstico relacionado aos transtornos mentais, e a realização de ações de redução de danos decorrentes do uso de crack, álcool e outras drogas. Porém, para que estes objetivos sejam alcançados torna-se necessário que o cuidado em saúde mental seja compartilhado entre as equipes de saúde e articulado entre as diferentes redes prioritárias do SUS.

É nessa perspectiva que a saúde da família necessita atuar por meio de ações de promoção em saúde e prevenção de doenças e agravos, contribuindo para modificar o modelo de saúde hegemônico assistencialista, biomédico e centrado na medicalização (Campos Júnior \& Amarante, 2015). Desta forma, é imprescindível a atuação ampliada e integrada da equipe da ESF, assumindo a corresponsabilidade pelo cuidado integral e longitudinal do indivíduo, considerando que a saúde mental e biológica não estão dissociadas, tampouco desarticuladas do contexto familiar-social-ambiental-cultural.

Frente a isso, a equipe de saúde que é constituída por profissionais de diversas formações em saúde, deve assumir a responsabilidade pelo cuidado dos usuários que apresentam transtornos mentais, levando em consideração os determinantes e condicionantes de saúde do território e de cada microrregião de saúde (Dutra \& Oliveira, 2015).

Conhecer as características dos usuários que apresentam diagnóstico de transtorno mental, é necessário para o planejamento de ações em saúde nos serviços de APS. Reis et. al. (2017) afirmam que para a efetivação de ações educativas e de promoção em saúde no território é preciso conhecer o panorama situacional da ESF e a demanda dos usuários, pois tal processo permite melhorar a oferta de ações em saúde, visando a qualificação do cuidado em saúde mental. 
Salienta-se que o município de origem do estudo possui em sua organização um modelo de atenção constituído de ESF e Unidades Básicas de Saúde (UBS) tradicionais; sendo que das 14 UBS apenas sete possuem função restrita de UBS tradicional, conforme demonstrado em estudo de Faria (2018). A justificativa da escolha deste território é devido a baixa cobertura de ESF do município, que é estimada em 22\% de acordo com o Plano Municipal de Saúde de 2018 -2021, sendo essa cobertura baixa para se atender às necessidades de saúde das microrregiões que constituem os territórios, visto que se tem uma demanda elevada da população que acessa os serviços de APS no município.

Tal situação interfere na qualidade do cuidado ao usuário que apresenta alterações psíquicas, pois a baixa cobertura no município se justifica devido a não organização da territorialização em saúde. A ESF em que o estudo foi realizado, atualmente, possui aproximadamente 3.600 usuários cadastrados neste território, de acordo com informações do sistema de informação em saúde utilizado pelo município (MV, 2021).

Desta forma, é primordial reconhecer os usuários atendidos nos serviços de APS, pois este processo contribui de maneira significativa para o planejamento de ações a serem desenvolvidas pela equipe na ESF. Diante disso, o objetivo deste estudo é caracterizar quais são os usuários com diagnóstico de saúde mental que acessam uma Estratégia Saúde da Família em um município do estado do Rio Grande do Sul.

\section{Metodologia}

Trata-se de um estudo transversal, descritivo, de abordagem quantitativa. Este tipo de estudo permite avaliar a população, em um determinado período do tempo, pois são de fácil realização, podendo ser utilizados na avaliação e planejamento da APS. Os estudos transversais são instrumentos importantes para descrever características de uma população, além de serem úteis no planejamento das ações de saúde (Estrela, 2018). A abordagem quantitativa permite formular hipóteses prévias, visando trazer à luz dados, indicadores, tendências observáveis no qual geram medidas confiáveis e sem vieses (Gerhardt \& Silveira, 2009.) Segundo Pereira et al. (2018), os dados quantitativos são analisados por meio de porcentagens, estatísticas e probabilidades, métodos numéricos, analíticos que são aplicados a determinado processo de investigação.

Os dados foram coletados a partir de informações secundárias, obtidas por meio de prontuários eletrônicos do sistema de informação em saúde utilizado pelos serviços de saúde do município, o qual fornece informações dos serviços próprios e conveniados. O município em questão integra a microrregião Verdes Campos da $4^{\mathrm{a}}$ Coordenadoria Regional de Saúde $\left(4^{\mathrm{a}}\right.$ CRS/RS) do Rio Grande do Sul, com população estimada de 280.505 habitantes para o ano de 2018 (IBGE, 2019).

Para a seleção dos prontuários que compuseram a amostra do estudo, solicitou-se aos Agentes Comunitários de Saúde (ACS) que atuam na ESF a lista de usuários em acompanhamento, que utilizam medicação controlada, denominadas listas dos usuários de benzodiazepínicos. A equipe utiliza a lista para identificar os usuários que apresentam transtornos de saúde mental no território e que estão em acompanhamento pela ESF.

Os critérios de inclusão do estudo foram: ser usuário de medicamento classificado benzodiazepínico e apresentar o nome na lista dos ACS. Os critérios de exclusão foram: prontuário incompleto (considerou-se incompleto o prontuário que apresentou somente $25 \%$ das informações), óbito do usuário, prontuário duplicado e/ou não encontrado no sistema de informação em saúde. A análise das informações obtidas através dos prontuários dos usuários abrangeu o período de novembro de 2015 a novembro de 2018.

A coleta dos dados foi realizada no mês de novembro de 2018, utilizando-se uma ficha organizada pelos autores do referido manuscrito, com as seguintes variáveis: sexo, idade, estado civil, com quem mora, escolaridade, diagnóstico de saúde mental, medicações utilizadas, usuário de substâncias psicoativas e percentual de informações disponíveis no prontuário, conformando um banco de dados no Programa Excel 2007 que, na sequência, passou por análise estatística descritiva. O projeto de pesquisa foi aprovado por um Comitê de Ética em Pesquisa, sob número de protocolo 3.030.422/2018, pautado nas diretrizes 
éticas da Resolução CNS n466/2012 em pesquisa com seres humanos, seja de proteção à privacidade ou identidade do sujeito e da instituição.

\section{Resultados}

A amostra inicial do estudo foi de 390 prontuários, que se refere ao total de pessoas listadas como usuários que fazem uso de benzodiazepínicos, residentes do território de abrangência da ESF. Destes, quatorze (3,6\%) foram excluídos pois os usuários foram a óbito, dois $(0,5 \%)$ não foram encontrados no sistema de informação em saúde, cinco (1,3\%) estavam duplicados nas listas e cento e três $(26,4 \%)$ foram excluídos tendo em vista que as informações nos prontuários estavam incompletas, não apresentando as informações necessárias para o estudo. Desta forma, a amostra final do estudo foi de 266 prontuários.

$\mathrm{Na}$ análise dos dados evidenciou-se que a média de idade dos usuários que apresentam transtornos de saúde mental em acompanhamento pela ESF era 59,07 anos $( \pm 14,65)$. Quanto ao sexo, 80,5\% dos usuários eram do sexo feminino e $19,5 \%$ do sexo masculino, conforme apresentado na Tabela 1.

Tabela 1: Sexo dos usuários de saúde mental.

\begin{tabular}{ll}
\hline Variável & $\mathrm{N}(\%)$ \\
\hline Sexo & \\
Feminino & $214(80,5)$ \\
Masculino & $52(19,5)$ \\
Total & $266(100)$ \\
\hline
\end{tabular}

Fonte: Elaboração dos autores (2018).

A Tabela 2 apresenta os diagnósticos de saúde mental que constavam nos prontuários dos pacientes. Com relação ao diagnóstico, verificou-se que em $136(51,12 \%)$ prontuários não constava a CID-10 (Classificação Internacional de Doenças). Dos 130 (48,87\%) prontuários que continham informações acerca da CID-10, 64 (22,22\%) usuários foram diagnosticados com depressão, $44(15,28 \%)$ com transtorno de ansiedade, $16(5,55 \%)$ com transtorno afetivo bipolar e $05(1,74 \%)$ com esquizofrenia. Identificou-se diagnósticos de "boderline", transtornos de humor (não especificados), síndrome do pânico, transtorno de personalidade, entre outros (Tabela 2). Destaca-se que o número total dos diagnósticos não coincide com o número total de prontuários analisados pelo fato que alguns destes apresentavam mais de um diagnóstico.

Tabela 2: Diagnósticos de Saúde Mental de pessoas residentes na área de abrangência de uma ESF.

\begin{tabular}{lc}
\hline \multicolumn{1}{c}{ Diagnóstico } & $\mathrm{N}(\%)$ \\
\hline Não Informado/Não Consta & $136(51,12)$ \\
Depressão & $64(22,22)$ \\
Transtorno de Ansiedade & $44(15,28)$ \\
Transtorno Afetivo Bipolar & $16(5,55)$ \\
Esquizofrenia & $5(1,74)$ \\
Transtorno de Humor & $6(2,08)$ \\
Outros & $17(5,90)$ \\
Total & $288(100)$ \\
\hline
\end{tabular}


A Tabela 3 apresenta dados sobre o estado civil, informações sobre o grupo familiar e sobre a escolaridade dos usuários com diagnóstico de saúde mental da ESF. Pode-se perceber que em 77,1\% dos registros nos prontuários não constavam dados referentes ao estado civil e $27,1 \%$ sobre a constituição do grupo familiar dos usuários. Identificou-se que 13,2\% dos usuários eram solteiros e 40,6\% moram com familiares e com o (a) companheiro (a). Quanto a escolaridade, 38,7\% tinham ensino fundamental incompleto (tabela 3).

Tabela 3: Estado civil, informações sobre grupo familiar e escolaridade dos usuários de saúde mental de uma ESF.

Variável

Estado civil

Solteiro(a)

Casado(a)

Divorciado(a)/separado(a)

União estável

Viúvo(a)

Não informado/Não constava

Com quem moram

Sozinho(a)

Com familiar

Com companheiro(a)

Com companheiro(a) e com familiar

Com amigos

Não informado/Não constava

Nível de escolaridade

Nenhum

Ensino Fundamental Incompleto

Ensino Fundamental Completo

Ensino Médio Incompleto

Ensino Médio Completo

Ensino Superior Incompleto

Ensino Superior Completo

Não informado/Não constava
$35(13,2)$

$20(7,5)$

$3(1,1)$

$1(0,4)$

$2(0,8)$

$205(77,1)$

$19(7,1)$

$108(40,6)$

$2(0,8)$

$72(27,1)$
$0(0,0)$

$10(3,8)$

$22(8,3)$

Fonte: Elaboração dos autores (2018). 
Na Tabela 4 se apresenta informações sobre o uso de psicofármacos pelos usuários de saúde mental da ESF. Com relação a essa informação, dos 266 prontuários analisados, 77,1\% faziam uso de antidepressivos e 50,4\% de benzodiazepínicos, 15,4\% utilizam dois antidepressivos diferentes, $1,5 \%$ dois anticonvulsivantes e 2,3\% dois antipsicóticos (Tabela 4).

Tabela 4: Uso de psicofármacos pelos usuários com transtornos de saúde mental.

\begin{tabular}{|c|c|}
\hline Classe Medicamentosa & $\mathrm{N}(\%)$ \\
\hline Benzodiazepínico & $134(50,4)$ \\
\hline Anticonvulsivante & $60(22,6)$ \\
\hline Antipsicótico & $38(14,3)$ \\
\hline Antidepressivos & $205(77,1)$ \\
\hline Sedativos & $0(0,0)$ \\
\hline Outra Classe & $34(12,8)$ \\
\hline \multicolumn{2}{|l|}{ Benzodiazepínico } \\
\hline Nenhum & $132(49,6)$ \\
\hline $\mathrm{Um}$ & $131(49,2)$ \\
\hline Dois & $3(1,2)$ \\
\hline \multicolumn{2}{|l|}{ Anticonvulsivante } \\
\hline Nenhum & $203(77,2)$ \\
\hline Um & $55(20,9)$ \\
\hline Dois & $4(1,5)$ \\
\hline Três & $1(0,4)$ \\
\hline \multicolumn{2}{|l|}{ Antipsicótico } \\
\hline Nenhum & $225(85,6)$ \\
\hline Um & $32(12,1)$ \\
\hline Dois & $6(2,3)$ \\
\hline \multicolumn{2}{|l|}{ Antidepressivos } \\
\hline Nenhum & $61(22,9)$ \\
\hline Um & $164(61,7)$ \\
\hline Dois & $41(15,4)$ \\
\hline
\end{tabular}

Fonte: Elaboração dos autores (2018).

$\mathrm{Na}$ Tabela 5 apresenta-se a quantidade de classes medicamentosas utilizadas pelos usuários. Percebe-se que 46,2\% utilizavam uma classe medicamentosa e 36,1\% duas classes (Tabela 5). 
Tabela 5: Quantidade de classes medicamentosas utilizadas por usuário.

\begin{tabular}{cc}
\hline Quantidade de Classes Medicamentosas & $\mathrm{N}(\%)$ \\
\hline Uma Classe & $123(46,2)$ \\
Duas Classes & $96(36,1)$ \\
Três Classes & $36(13,6)$ \\
Quatro Classes & $7(2,6)$ \\
Cinco Classes & $4(1,5)$ \\
\hline
\end{tabular}

Fonte: Elaboração dos autores (2018).

Em relação ao uso de drogas ilícitas ou substâncias psicoativas, 3\% dos usuários do território da ESF faziam uso, sendo que $1,5 \%$ utilizavam cocaína, $0,4 \%$ utilizavam maconha e cocaína e 1,1\% dos prontuários não continham a informação da droga utilizada. Dos prontuários analisados 9,4\% apresentaram informações completas, $81,9 \%$ continham $75 \%$ das informações e 8,6\% registraram $50 \%$ das informações.

\section{Discussão}

A pesquisa revelou que os prontuários analisados não apresentavam informações necessárias para o acompanhamento dos usuários de saúde mental, demonstrando fragilidades na gestão do cuidado. Neste sentido Teixeira et. al. (2017) apontam que o prontuário eletrônico é um documento que contempla o conjunto de informações de saúde do usuário e os procedimentos. Deve-se registrar a descrição da assistência prestada, a anotação dos sinais vitais, sintomas, resultados de exames, entre outros, que incluem a história clínica do usuário e os encaminhamentos aos demais serviços de saúde da rede (Ribeiro, et. al., 2018).

Cabe destacar que a Carta dos Direitos dos Usuários da Saúde (Brasil, 2011) prevê o "registro atualizado e legível no prontuário" de todas as informações relativas à condição de saúde, abrangendo os procedimentos aos quais o usuário foi submetido, às avaliações, motivo do atendimento e demais informações necessárias para avaliação. Além disso, o prontuário tem caráter legal, sigiloso e científico, possibilitando registrar informações relacionadas à saúde dos usuários do SUS, permitindo a comunicação entre a equipe multiprofissional e a comunicação intersetorial, bem como o percurso nos diferentes pontos da RAS que o usuário transita, possibilitando, desta maneira, a integração dos serviços de saúde e a garantia da coordenação do cuidado (Rodrigues, et. al., 2017.)

Versani et. al. (2015) reforçam o papel do registro das informações como forma de comunicação, permitindo avaliar a qualidade do atendimento ofertado aos usuários e a organização intersetorial, oferecendo para a equipe informações referentes ao acesso do usuário nos diferentes serviços de saúde. Os prontuários eletrônicos são ferramentas que auxiliam na qualificação do processo de trabalho, tornando-se necessário para o planejamento, por parte da gestão municipal de saúde no que se refere a adesão, seja via empresa pública ou privada; e a avaliação, junto aos trabalhadores do SUS, quanto a qualidade dos registros, contribuindo para o desenvolvimento de ações de educação permanente com os sujeitos envolvidos neste processo (Cardoso, et. al., 2017).

Em relação à variável sexo, os resultados vão ao encontro do estudo de Campos, Ramalho e Zanello (2017), que constataram que 72,2\% dos usuários de saúde mental, de um Centro de Atenção Psicossocial - CAPS II (que acompanham usuários com transtornos mentais e/ou egressos de hospitalizações em instituições psiquiátricas) eram do sexo feminino. No que se refere aos CAPS-AD (que acompanha usuários de álcool e outras drogas), o sexo predominante é masculino (Silva, et. al., 2017). Para Zanetti et. al. (2017) os transtornos mentais são prevalentes em mulheres pelo fato destas estarem suscetíveis a 
ansiedade e depressão decorrentes do período reprodutivo e porque elas reconhecem e relatam os sintomas com mais frequência nos serviços de APS.

No estudo em questão, os diagnósticos prevalentes foram Depressão e Transtorno de Ansiedade, semelhante ao encontrado em estudo de Oliveira, Jorge e Mariotti (2017) que identificou a depressão como o diagnóstico mais frequente. A depressão afeta um quantitativo expressivo da população, sendo descrito como o transtorno psiquiátrico mais prevalente em todo mundo, manifestando-se de maneira diferente entre os sexos e sendo frequente entre as mulheres (Cardoso, 2011; Costa et. al., 2017). No que se refere ao diagnóstico de ansiedade, Bezerra et. al. (2017) destacam que, comumente, há uma associação entre insônia e ansiedade, e que esta associação tem uma prevalência, no Brasil, de 12\% a 76\% da população, o que demonstra sua relevância.

O estudo de Freitas et. al. (2017) identificou que 54,5\% dos usuários de um CAPS em Rondônia eram solteiros. Porém Oliveira, Jorge e Mariotti (2017) encontraram resultados diferentes, em que 56,4\% dos usuários de saúde mental de um CAPS II eram casados. Analisar este dado é importante, visto que, conforme Dutra e Oliveira (2015), os usuários com questões de saúde mental têm dificuldade de criar redes sociais fortes e comumente estes laços sociais apresentam-se fragilizados ou rompidos, por questões sociais, econômicas, políticas ou psicológicas.

Evidenciou-se que 38,7\% dos usuários possuíam o ensino fundamental incompleto, dados estes que vão ao encontro do estudo de Oliveira, Jorge \& Mariotti (2017), que identificou que em um CAPS II, os usuários não haviam concluído o ensino fundamental. A baixa escolaridade de usuários de saúde mental foi encontrada em estudo de Silva et. al. (2015), no qual 84,4\% dos usuários de benzodiazepínicos na APS possuem o ensino fundamental. Zanetti et. al. (2017) destacam que a baixa escolaridade está frequentemente associada com o diagnóstico de depressão.

No estudo realizado identificou-se que os psicofármacos mais utilizados pelos usuários de saúde mental foram os antidepressivos seguidos dos benzodiazepínicos. Prado, Francisco e Barros (2017) encontraram, em seus estudos, resultados semelhantes aos achados nesta pesquisa, em que os usuários utilizavam antidepressivos, seguidos de benzodiazepínicos. Borges, Hegadoren e Miasso (2015) identificaram resultados similares, onde os psicofármacos prescritos para a população do estudo com frequência foram antidepressivos, seguidos de benzodiazepínicos.

Zanetti et. al. (2017) destacam que o uso de antidepressivos vem crescendo mundialmente e que o maior consumo dessa classe medicamentosa ocorre em mulheres entre 35 e 64 anos. $O$ uso expressivo de benzodiazepínicos verificado no presente estudo pode estar relacionado com a disponibilidade da medicação pelo SUS. Para Silva et. al. (2015), o uso frequente de psicofármacos é de grande incidência, sendo o Brasil um dos maiores importadores de Diazepam (benzodiazepínico) do mundo. Oliveira, Mota e Castro (2015) destacam que o uso indiscriminado de benzodiazepínicos pode estar relacionado com a facilidade em adquirir estes medicamentos, o baixo custo, a inadequada indicação e distribuição gratuita pelo governo, entre outros, colaborando para que esse uso desenfreado se torne um problema de saúde pública.

Nos prontuários analisados neste estudo, a informação sobre o uso de drogas ou substâncias psicoativas foi baixa, demonstrando que este não se configura, segundo o registro oficial dos prontuários, um problema de saúde no território estudado. Entretanto, o não registro não assegura o fato de que no território as pessoas não façam uso das mesmas, pelo fato destas substâncias, na sua maioria, serem ilegais. Para Rocha et. al. (2015) o uso de álcool, maconha, cocaína, crack, entre outras, pode estar relacionado com a busca pelo prazer ou a tentativa de fuga da realidade. Porém, quando essas substâncias são utilizadas por indivíduos com problemas de saúde mental, evidencia-se diversos efeitos, tais como potencialização dos transtornos mentais existentes, desencadeamento de comorbidades, dificuldades de adesão ao tratamento, comprometendo as relações pessoais e sociais do usuário.

Cabe destacar que a falta de informações nos prontuários dos usuários pode comprometer a coordenação do cuidado, um dos principais atributos da APS, uma vez que a informação é essencial para o diagnóstico, o tratamento e as orientações 
prestadas para o cuidado do usuário (Carrer, et. al., 2016). Por fim, a falta de informações de saúde prejudica a construção do Projeto Terapêutico Singular, que é uma ferramenta de cuidado em saúde mental que possibilita a articulação de diferentes serviços da RAPS e considera as necessidades específicas do usuário e de sua rede de apoio (Melo, et. al., 2017).

\section{Conclusão}

$\mathrm{O}$ estudo indicou que os prontuários analisados não estavam completamente preenchidos, limitando o conhecimento dos usuários com questões relacionadas à saúde mental do território. Essa fragilidade aponta para a necessidade de estabelecimento de estratégias de educação permanente em saúde, tornando visível à equipe multiprofissional, a importância do preenchimento correto do prontuário, sendo que ao mantê-lo atualizado, poderá contribuir para a efetivação de ações e intervenções de saúde mental no território.

A depressão foi o diagnóstico de saúde mental prevalente entre as mulheres com baixa escolaridade, de núcleos familiares pequenos, condição esta que atualmente conforma-se em um importante problema de saúde pública no SUS. A ansiedade também foi uma condição prevalente no estudo, sendo que muitos usuários procuram a APS para realizar o tratamento destas e de outras desordens psíquicas, realidade que requer preparo dos serviços de saúde e de seus profissionais.

Compete à gestão municipal e aos Núcleos de Educação Permanente em Saúde atuar por meio de estratégias de qualificação das equipes multiprofissionais, priorizando um cuidado humanizado, resolutivo e integral aos usuários de saúde mental, visando o registro completo das informações nos prontuários. Reforça-se que a APS tem função central na coordenação do cuidado e ordenação das redes, assim é preciso uma relação compartilhada entre todos os serviços da RAS, de forma a garantir a articulação entre os entes federativos e promover um cuidado alinhado às necessidades de saúde da população. Sugere-se a realização de pesquisas futuras nestes serviços de APS, visto que alguns processos como a implementação da Planificação da Atenção à Saúde, o financiamento da APS e atuação das Equipes de Atenção Primária são questões que passaram por mudanças e que irá impactar de forma direta no cuidado do usuário com diagnóstico de saúde mental, assim como a própria implementação da Portaria 3.588 de 2017 o que se percebe como retrocesso na saúde mental.

\section{Agradecimentos}

Agradecemos à equipe de Saúde da Família, cenário no qual o estudo foi realizado, em especial aos Agentes Comunitários de Saúde e à Enfermeira do serviço, visto que estes contribuíram de forma significativa na pesquisa. Os autores agradecem a residência Multiprofissional em Sistema Único de Saúde - área de concentração Atenção Básica / Estratégia de Saúde da Família da Universidade Federal de Santa Maria (UFSM - RS) pela oportunidade e pelas experiências proporcionadas durante o período de realização da residência no qual proporcionou a execução deste estudo.

\section{Referências}

Bezerra, E., Alves Júnior, E., Diniz, A., Alves, L., Felício, I., \& Nóbrega, R. (2017). Utilização de benzodiazepínicos por usuários do sistema único de saúde. Biofarm, 13 (3), 17-21.

Borges, T., Hegadoren, K., \& Miasso, A. (2015). Transtornos mentais comuns e uso de psicofármacos em mulheres atendidas em unidades básicas de saúde em um centro urbano brasileiro. Rev Panam Salud Publica, 38 (3), 195-201.

Ministério da Saúde. Carta dos direitos dos usuários da saúde: Ministério da Saúde.

Campos, I., Ramalho, W., \& Zanello, V. (2017). Saúde mental e gênero: O perfil sociodemográfico de pacientes em um centro de atenção psicossocial. Estudos de Psicologia, 22 (1), 68-77.

Campos Junior, A., \& Amarante, P. (2015). Estudo sobre práticas de cuidado em saúde mental na Atenção Primária: o caso de um município do interior do estado do Rio de Janeiro. Cadernos Saúde Coletiva, 23 (4), 425-435.

Cardoso, L. (2011). Psicoterapias comportamentais no tratamento da depressão. Psicologia Argumento, 29 (67), $479-489$. 
Cardoso, R., Ferreira, B., Martins, W., \& Paludeto, S. (2017). Programa de educação permanente para o uso do prontuário eletrônico do paciente na enfermagem. J Health Inform, 9 (1), 25-30.

Carrer, A., Toso, B., Guimarães, A., Conterno, J., \& Minosso, K (2016). Efetividade da Estratégia Saúde da Família em unidades com e sem Programa Mais Médicos em município no oeste do Paraná, Brasil. Ciência \& Saúde Coletiva, 21 (9), 2849-2860.

Costa, T., Medeiros, R., Sousa, M., Uchida, R., \& Miranda, F. (2017). Intensidade e sintomas depressivos em usuários da estratégia saúde da família. Interfaces Científicas - Saúde e Ambiente, 5 (3), 47-56.

Dutra, V. D., \& Oliveira, R. P. (2015). Revisão integrativa: as práticas territoriais de cuidado em saúde mental. Aquichan, 15 (4), $529-540$.

Estrela, C. (2018). Metodologia Científica: ciência, ensino, pesquisa. (3 ${ }^{\mathrm{a}}$ ed.): Artes Médicas.

Faria, R. (2018). A territorialização da atenção primária à saúde do sus: avaliação dos resultados práticos implementados numa cidade da região sul do Brasil. Hygeia, 14 (29), 89-104.

Freitas, B., Matos, C., Silva, P., Santos, J., \& Batista, E. (2017). Profile of users diagnosed with schizophrenia of a CAPS in the countryside of Rondonia. Nucleus, 14 (1), 41-54.

Gerhardt, T. E., \& Silveira, D. T. (2009). Métodos de pesquisa: Editora da UFRGS.

Instituto Brasileiro de Geografia e Estatística. https://www.ibge.gov.br/.

Melo, T., Weinert, L., Hoshino, M., \& Maldaner, T. (2017). Perfil dos usuários de serviços de reabilitação no sistema único de saúde. Revista da Universidade Vale do Rio Verde, 15 (2), 65-77.

MV (2021). Prontuário Eletrônico do SOUL MV. MV Informática Nordeste Ltda. 2015. <http://www.mv.com.br/pt/>

Oliveira, A., Jorge, I., \& Mariotti, M. (2017). Perfil sociodemográfico de usuários de um centro de atenção psicossocial e o trabalho. Cadernos Brasileiros de Terapia Ocupacional, 25 (4), 795-802.

Oliveira, E., Medeiros, A., Trajano, F., Chaves Neto, G., Almeida, S., \& Almeida, L. (2017). Mental health care in the territory: conceptions of primary health care professionals. Escola Anna Nery, 21 (3), 1-7.

Oliveira, J., Mota, L., \& Castro, G. (2015). Uso indiscriminado dos benzodiazepínicos: a contribuição do farmacêutico para um uso consciente. Transformar, (7), 214-226.

Oliveira, M., Cestari, T., Pereira, M., Pinho, P., Gonçalves, R., \& Claro, H. (2014). Processos de avaliação de serviços de saúde mental: uma revisão integrativa da literatura. Saúde em Debate, 38 (101), 368-378.

Pereira, A. S., Shitsuka, D. M., Parreira, F. J., \& Shitsuka, R. (2018). Metodologia da pesquisa científica. [e-book]. Santa Maria. Ed. UAB/NTE/UFSM. https://repositorio.ufsm.br/bitstream/handle/1/15824/Lic_Computacao_Metodologia-Pesquisa-Cientifica.pdf?sequence=1 .

Prado, M., Francisco, P., \& Barros, M. (2017). Uso de medicamentos psicotrópicos em adultos e idosos residentes em Campinas: um estudo transversal de base populacional*. Epidemiologia e Serviços de Saúde, 26 (4), 747-758.

Reis, L., Pereira, S., Teixeira, C., Cardoso, L., \& Gherardi-Donato, E. (2017). Perfil de usuários diagnosticados com deficiência intelectual atendidos em um serviço ambulatorial de saúde mental. Cadernos Brasileiros de Saúde Mental, 9 (23), 39-50.

Ribeiro, W., Andrade, M., Flach, D., Santana, P., Souza, D., \& Almeida, V. (2018). Implementação do prontuário eletrônico do paciente: um estudo bibliográfico das vantagens e desvantagens para o serviço de saúde. Revista Pró-univerSUS, 9 (1), 7-11.

Rocha, F., Oliveira, R., Brum, D., Cavalcante, R., \& Machado, R. (2015). Epidemiologia dos transtornos do desenvolvimento psicológico em adolescentes: uso de álcool e outras drogas. Revista da Rede de Enfermagem do Nordeste, 16 (1), 54-63.

Rodrigues, P. M., Paraboni, P., Arpini, D., Brandolt, C., Lima, J., \& Cezar, P. (2017). O registro em prontuário coletivo no trabalho do psicólogo na Estratégia Saúde da Família. Estudos de Psicologia. 22 (2), 195-202.

Sampaio, J., Guimarães, J., Carneiro, C., \& Garcia Filho, C. (2011). O trabalho em serviços de saúde mental no contexto da reforma psiquiátrica: um desafio técnico, político e ético. Ciência \& Saúde Coletiva, 16 (12), 4685-4694.

Silva, D., Torrezan, M., Costa, J., Garcia, A., \& Toledo, V. (2017). Perfil sóciodemográfico e epidemiológico dos usuários de um centro de atenção psicossocial álcool e drogas. Revista de Enfermagem e Atenção à Saúde, 6 (1), 67-79.

Silva, M., Aguiar, M., \& Moreira, T. (2016). Entre os nós da rede de saúde mental: as práticas de agentes comunitários de saúde. Revista Baiana de Saúde Pública, 40 (3), 713-728.

Silva, V., Botti, N., Oliveira, V., \& Guimarães, E. (2015). Perfil epidemiológico dos usuários de benzodiazepínicos na atenção primária à saúde. R Enferm Cent O Min, 5 (1), 1393-1400.

Teixeira, A., Longen, W., Minetto, A., \& Pacheco, R. (2017). Revisão de registros dos prontuários no setor de fisioterapia das clínicas integradas UNESC. Inova Saúde, 6 (2), 98-114.

Versani, C., Ohara, C., Saparolli, E., Silva, P., Amaral, E., \& Siqueira, L. (2015). Avaliação da qualidade dos registros das fichas "C" de crianças menores de 01 ano das equipes de saúde da família de Montes Claros/MG. Revista de APS, 18 (1), 6-15. 
Research, Society and Development, v. 10, n. 1, e37210111909, 2021

(CC BY 4.0) | ISSN 2525-3409 | DOI: http://dx.doi.org/10.33448/rsd-v10i1.11909

Zanetti, L., Stumm, E., Bosse, F., Oliveira, R., Bandeira, V., \& Colet, C. (2017). Tratamento medicamentoso e não medicamentoso de usuários de um Centro de Atenção Psicossocial. Scientia Médica, 27 (4), 1-10. 